\title{
Reinforcement-test-blank acquisition programming under the unmixed-list design in paired-associate learning ${ }^{1}$
}

CHIZUKO IZAWA, State University of New York at Buffalo, Buffalo, N.Y. 14226

Two experiments, with 64 university students, were conducted under the ummixed-list design with larious repetitive reinforcement $(R$ )-test $(T)$-blank $(B)$ acquisition programming: Conditions $R T$, $R T T, R T B$, and $R B T$. The major empirical findings did not differ, generally, from those obtained under the mixed-list design, indicating that the main effects overcame procedural artifacts embedded in the mixed-list design. Other things being equal, the learning of verbal pairs was a function of $R-T \cdot B$ acquisition programming. Unreinforced test trials in paired-associate learning prevented retention losses and, at the same time, increased the conditioning power of the later reinforcement trials.

Acquisition programming of reinforcement (R) and test (T) trials in paired-associate learning was recently introduced as a useful new experimental variable for investigating processes underlying the learning of verbal pairs (Izawa, 1966), in which $\mathrm{R}$ and $\mathrm{T}$ trials are, respectively, defined as paired presentations of both stimulus and response terms of a given pair and presentations of the stimulus term alone without any feedback from $E$.

Further, by replacing some of the unreinforced $T$ trials by blank (B) trials, during which nothing was presented, or by neutral (N) trials, during which colored geometrical figures were presented to be named by Ss, hidden functions of tests in paired-associate learning were brought to light (Izawa, 1967a). In further pursuing the effects of $\mathrm{T}$ trials, however, a mixed-list design used in the study raised a procedural problem.

Under a mixed-list design, a group of Ss learn a single list with multiple conditions, whereas under an unmixed-list design, each of the several groups of Ss learns a list with a unitary condition. The effects of these two list designs were reported, on one hand, to be different, as in Wright (1967) and Johnson \& Penney (1966), Wickens \& Cermak (1967) and Postman (1966), and Reynolds (1964), respectively, in terms of acquisition, transfer effects, and methods (anticipation vs nonanticipation). On the other hand, no differences were demonstrated between the two designs as in Underwood \& Schulz (1960), Birnbaum (the main effects, 1968), and Twedt \& Underwood (1959), respectively, in terms of acquisition and transfer effects.

When acquisition sequences were programmed exclusively of $\mathrm{R}$ and $\mathrm{T}$ trials, no differential results were obtained between mixed-and unmixed-list designs as in Izawa (1967b). However, the same results may not hold when $B$ or $N$ trials are introduced in acquisition sequences. Take, for example, a highly simplified experiment with two conditions learned with the following repetitive sequences:

$$
\begin{aligned}
& \text { (j) } \\
& 1=3+567 \times 4.5 \\
& \text { conditink RT R T R T R T..... }
\end{aligned}
$$

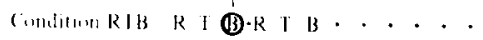

When this experiment is conducted under a mixed-list design, one-half of the pairs within the list will be learned with the RT sequence and the other half with the RTB sequence. Consider the effects of the $B$ trial of Condition RTB on Cycle 3. The B trial, undoubtedly, generates effects upon the R trial of Condition RTB on Cycle 4 as well as its subsequent trials (as indicated by a horizontal arrow). However, since the list is mixed, perhaps the more pronounced effects of the $B$ trial are likely to be produced upon the $\mathbf{R}$ trial of Condition RT on Cycle 3 (as shown by a vertical arrow). This follows from the fact that the items assigned to the B trial of Condition RTB, being replaced by blanks, are not shown on that cycle, and that only one-half of the list assigned to Condition RT is presented as pairs (Rs) on Cycle 3. This operation may give undue advantage to Condition RT, making it difficult to compare the two conditions. The results contain an artifact based on a mixed-list design.

Such artifacts may be entirely eliminated when an unmixed-list design is used. To insure that the influences of $B$ trials are placed upon the subsequent trials of their own condition but not upon those of other conditions, the present two experiments were conducted under the unmixed-list design with major conditions selected from Izawa (1967a), which was run under a mixed-list design.

\section{METHOD}

The repetitive experimental sequences used in two experiments were as follows

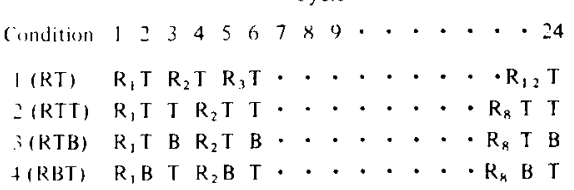

Condition names may be given in terms of its basic replication, defined as a unit of repetition pattern. On an $R$ trial, both stimulus and response terms of a pair were presented for $S$ to read aloud. On a $T$ trial, only the stimulus term was presented for him to supply its corresponding response term orally, with no feedback from E. On a B trial, nothing was presented, but neither talking nor getting up were allowed. Exposure times for $R, T$, and $B$ trials presented on a Stowe Memory Drum, B-539, were all $4 \mathrm{sec}$ each.

Twenty CVC-two-digit-number pairs and 20 CVC-noun pairs were learned in Experiments 1 and 2, respectively, both lists being adopted from Izawa (1967a). The presentation orders of the items within $R$ and $T$ cycles were, respectively, randomized. Ss were instructed to learn the pairs as fast as possible.

Sixty-four students (32 in each experiment), hired at the University of California at Berkeley, participated as Ss. With the unmixed-list procedure, four groups of eight sach learned the 20 pairs under the assighed acquisition sequence within each experiment. A practice task with an identical sequence for all Ss, which preceded the main PAL task, showed no group differences in each experiment.

\section{RESULTS AND DISCUSSION}

In Condition RTT, no significant changes were observed from the first $T$ to the second following each $\mathrm{R}$ at any stager of acquisition in each experiment, indicating that neither learning nor forgetting, with respect to response probabilities, occurred on unreinforced test per se.

Since no differences were obtained over the successive $T s$ in each replication, learning curves in terms of error proportions (including omissions) were drawn as a function of the preceding reinforcements with respect to the first $T$ following each $\mathrm{R}$ in Fig. 1 . When tested with respect to the total errors per $S$ on the first $T s$ following the first eight $R s$ in Fig. 1, learning curves differed significantly within each experiment: $F(3,28)=93.61$, $\mathrm{p}<.001$ in Experiment 1, and $F(3,28)=2.90, p \doteq .05$ in Experiment 2 . The findings suggest that, other things being equal, learning is a function of acquisition programming.

In both experiments, Condition RTT, with two Ts per replication, clearly excelled Condition RT with one $\mathrm{T}$ per replication. As for the conditions that had 


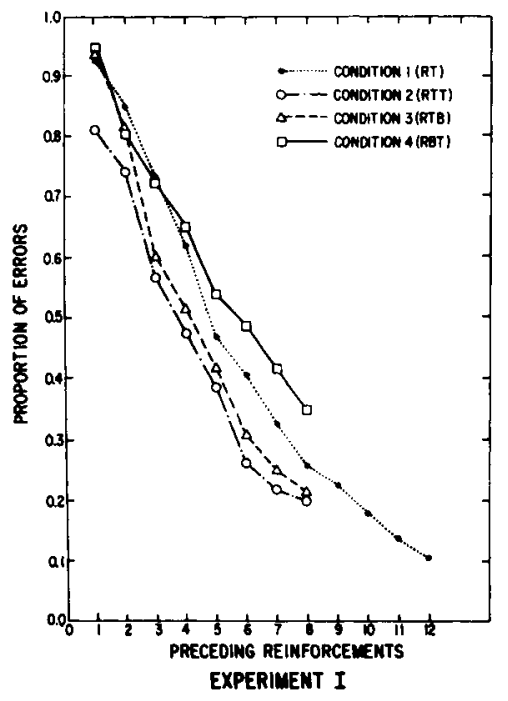

the identical time intervals between each $R$ as Conditions RTT, RTB, and RBT; if unreinforced $\mathrm{T}$ trials have their unique functions as compared to mere lapse of time and possible S's oriented covert rehearsals on B trials, Condition RTT should show substantial differences from the others. This expectation is fairly well borne out: Although the differences between Conditions RTT and RTB in Experiment 1 were not necessarily large, all of the other differences were significant in both experiments. Evidently, the more tests, the better the learning. The underlying process behind these empirical phenomena is that unreinforced $\mathrm{T}$ trials in paired-associate learning facilitate acquisition by potentiating the effectiveness or the conditioning power of the subsequent $\mathbf{R}$ trials.

In Experiment 2, Condition RBT showed an inferior performance early in acquisition and yet produced a superior performance at the end, i.e., the crossing of the RBT curve with the others was demonstrated. Although the same phenomenon was not observed exactly in Experiment 1, there were some indications of a similar trend. Excluding the first three data points in Fig. 1, the relatively early inferior performance of Condition RBT was demonstrated. Judging from the steepest slope of the curve as compared to the relatively milder slopes of the others, if acquisition had been continued further in Experiment 1, close to the mastery of the list, it can be inferred that the similar crossing could have occurred. In particular, it is interesting to note the manifestation of nonmonotonic differences between Conditions RTB and RBT during the course of acquisition, which depended on their programming, despite the fact that both experienced identical numbers of $R$, $\mathrm{T}$, and $\mathrm{B}$ trials.

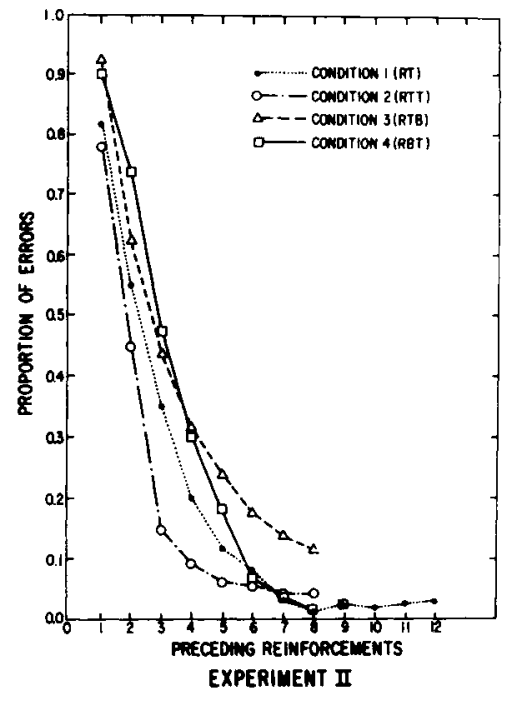

The total overt errors made in Conditions 1 (RT), 2 (RTT), 3 (RTB), and 4 (RBT) were, respectively, 334, 701, 130, and 441 in Experiment 1 and 76, 134,75, and 123 in Experiment 2. The ratios of the overt errors to the total incorrect responses for the respective conditions were $39.11 \%$, $59.39 \%, 18.95 \%$, and $51.94 \%$ in Experiment 1 and $20.87 \%, 24.10 \%$, $15.81 \%$, and $28.01 \%$ in Experiment 2 . Obviously, Ss attempted more guesses when the response terms were numbers (Experiment 1) than when they were words (Experiment 2). The majority of overt errors, 63\%-72\% in Experiment 1 and $60 \%-89 \%$ in Experiment 2, occurred only once per item. The absolute total of the numbers of repeated errors per item was small, with no sign of stereotypes.

The major empirical findings obtained under the present unmixed-list design were the direct confirmation of those observed under a mixed-list design (Izawa, 1967a), with a minor deviation that the differences between Conditions RTT and RTB were larger in Experiment 2 than were those in the previous study. The same differences, however, were insignificant in Experiment 1. Overall, both mixed-and unmixed-list designs produced basically the same empirical outcomes. It is possible that the procedural artifacts based on the mixed-list design were negligible, as demonstrated by Izawa (1967b), when the acquisition sequences consisted only of $R$ and $T$ trials, and in other learning situations, such as in Underwood \& Schulz (1960) and Twedt \& Underwood (1959). However, because of the opposing evidence that mixed- and unmixed-list designs produced differential results, as in Wickens \& Cermak (1967), Wright (1967), Postman (1966), Johnson \& Penney (1966), and Reynolds (1964), and because of the overwhelming logical possibility of the
Fig. 1. Learning curves in terms of the first $T$ trial following each $R$ trial.

procedural artifacts of mixed-list design when B trials are involved, as discussed earlier, it seems more reasonable to interpret the effects of R-T-B acquisition programming upon learning and the functions of unreinforced tests as being too powerful to be masked by the probable artifacts embedded in the mixed-list design.

Thus, with clear evidence obtained under the present unmixed-list design, it may be safely concluded that, other things being equal, the learning of verbal pairs is a function of R-T-B acquisition programming. Unreinforced test trials in paired-associate learning have two different functions. First, from the constancy demonstrated in response probabilities over the successive Ts per replication in Condition RTT, T trials may be concluded to prevent retention losses. Sicond. from learning-curve analyses showing that the more $\mathrm{T} s$, the less the errors, unreintorced tests may be inferred to potentiate the effectiveness of the subsequent reinforced trials in paired-associate learning.

\section{REFERENCES}

BIRNBAUM, I. M. Response-class similarity and first-list recall with mixed and unmixed transfer designs. Journal of Experimental Psychology, 1968, 77. 542-546.

IZAWA, C. Reinforcement-test sequences in paired-associate learning. Psychological Reports, 1966, 18, 879-919.

IZAWA, C. Function of test trials in paired-associate learning. Journal of Experimental Psychology, 1967a, 75, 194-209.

IZAWA, C. Mixed- versus unmixed-list designs in paired-associate learning. Psychological Reports, 1967b, 20, 1191-1200.

JOHNSON, G. J., \& PENNEY, R. K. The effect of mixed vs. unmixed list designs in paired-associatc learning. Journal of Verbal Learning \& Verbal Behavior, 1966, 5, 234-239.

POSTMAN, L. Differences between unmixed and mixed transfer designs as a function of paradigm. Journal of Verbal Learning \& Verbal Behavior, 1966. 5, 240-248.

REYNOLDS, J. H. Effects of non-anticipation and anticipation procedures upon paired-associate learning in unmixed and mixed list designs. Psychological Reports. 1964, 15, 795-801.

TWEDT, H. M., \& UNDERWOOD, B. J. Mixed vs. unmixed lists in transfer studies. Journal of Experimental Psychology, 1959, 58, 111-116. UNDERWOOD, B. J., \& SCHULZ, R. W. Response dominance and rate of learning paired-associates. Journal of General Psychology, 1960, 62, 153-158.

WICKENS, D. D., \& CERMAK, L. S. Transfer effects of synonyms and antonyms in mixed and unmixed lists. Journal of Verbal Learning \& Verbal Behavior, 1967, 6, 832-839.

WRIGHT, J. H. Effects of stimulus meaningfulness, method of presentation, and list design on the learning of paired-associates. Journal of Experimental Psychology, 1967, 73, $72-77$. 
NoT

I. This study is supported in part by is postatostoral tellowship at the Institute of Human I caming. Iniversity of (alifornia. Berkeley. and in part by (irant WH $12979-1 / 2$ at the Vational Institute of Mental Health. The author is gratciul to l'rot. Leo P'ostman for his asvistance in facilitating this research. Many thanks are due to I lainc $N$. Blesi who collected linc data.

\section{Attitudinal effects of mere exposure:}

\section{A replication and extension ${ }^{1,2}$}

\author{
MICHEL PIERRE JANISSE, University of \\ Waterloo, Waterlso, Ont., Canado
}

Three sources of stimulus input. one visual and two aural, were distinguished in Zajomc's (1968) study that demonstrated that rated "goodness"-of-meaning of nonsense words is a positive function of exposure frequency. In the present study, both a replication condition and an experimental condition that deleted one of the aural sources of input vielded the same function $(p<025)$. In the replication condition. the stimuli were rated somewhat more "goed" than in the experimental condition $(p<.087)$, indicating that the increased aural input enhanced the ratings. Pessible areas of fiture research were mentioned.

In a 1968 monograph, Zajonc suggested, and presented evidence for, the hypothesis that repeated exposure to a stimulus enhances a S's attitude toward that stimulus. In this context, to enhance means to be rated higher on a "goodness"-of-meaning scale. In one study, 12 seven-letter nonsense words were presented at six different frequencies from 0 to 25 . As each word was shown, $\mathrm{S}$ would pronounce it after $E$. When asked subsequently to rate each word on the "goodness"-of-meaning scale, Ss tended to rate a word higher as a function of its frequency of exposure. At each presentation of a word, $S$ was exposed to the stimulus in three ways: seeing it, hearing $E$ pronounce it, and hearing himself pronounce it. If one of these three types of exposure were eliminated, Zajonc's hypothesis would predict that $\mathrm{S}$ would show less of an enhancement to the stimulus, since he would be deprived of one source of input, reducing frequency of exposure by one-third. Ruling out S's pronunciation would not necessarily eliminate a subvocal pronunciation, still leaving three types of exposure at each presentation. Therefore, in the present study, an experimental condition deleted

\section{E's pronunciation of the nonsense word. METHOD}

Twenty-four female registered nurses at the Hamilton Psychiatric Hospital, Hamilton, Ontario, served as Ss in the present experiment. In the replication condition, 12 Ss were presented with the stimulus nonsense words, as in Zajonc's study. They were told that each word was a Turkish adjective, and that they were to look at it and pronounce it after $E$. In the experimental condition, the $12 \mathrm{Ss}$ were told just to look at each word and pronounce it. E did not pronounce it.

In both conditions, two nonsense words appeared at each exposure frequency $(0,1$, $2,5,10$, and 25$)$. The 12 stimuli appeared an equal number of times at each frequency level under each condition. Six different combinations of stimuli and exposure frequencies were employed, each being used with two Ss per condition. Stimuli were presented on $3 \times 5$ in. index cards and were shuffled before presentation to each $\mathrm{S}$.

After the stimulus presentations, $S$ was told that her task was to guess what each word meant in Turkish, but that since this was quite difficult, it was suggested instead that she rate the "goodness" or "badness" of the word's Turkish meaning. The rating was done on a 7-point, good-bad, Likert type of scale. The order of presentation of stimulus words for rating was randomized for each $\mathrm{S}$. Two additional stimuli, never seen by $S$ (the 0 frequency presentation), were also rated.

Following these procedures, each $S$ was told the true nature of the experiment, was thanked for her participation, and was asked not to reveal, for a reasonable length of time, the details of the task.

\section{RESULTS}

Figure 1 presents the ratings of "goodness"-of-meaning across each of the six exposure frequencies for the replication ( $E$ and $S$ pronounce) condition and the experimental (S pronounce) condition. Analysis of variance indicated that the exposure-frequency effect was significant
$(F=2.72, \mathrm{df}=5 / 110, \mathrm{p}<.025)$, and that the difference between the replication and experimental conditions approached significance $(F=3.14, \quad d f=1 / 22$, $\mathrm{p}<.087$ ).

Ten of the 12 stimulus nonsense words were rated as meaning something "better" when they appeared at high-frequency levels (i.e., 5, 10, and 25) than when they appeared at low-frequency levels (i.e., 0,1 , and 2). The chance of this occurring in 10 or more stimulus words has a probability of less than .002. An overall $t$ test between the ratings of high- and low-frequency stimulus words indicated that the high-frequency words were seen as significantly "better" $(\mathrm{t}=3.34, \mathrm{df}=286$, $\mathrm{p}<.005)$. Nine of the 12 stimulus words were rated higher in the replication condition than in the experimental condition. This approaches significance $(p<.073)$.

\section{DISCUSSION}

The demonstration of the exposure-frequency effect in the present study is consistent with the findings of Zajonc (1968) and others (e.g., Becknell, Wilson, \& Baird, 1963; Johnson, Thomson, \& Frinke, 1960). In subsequent studies using Chinese-like characters and yearbook photographs of men's faces, where pronunciation by either $E$ or $S$ was impossible, Zajonc demonstrated that visual input alone is sufficient to produce the effect. However, the average ratings in the three studies were not compared with each other as in the two conditions of the present study. The results here indicate both that the effect may be obtained with nonsense-word stimuli when $E$ remains silent, and that the effect tends to appear lower on the "goodness"-of-meaning scale

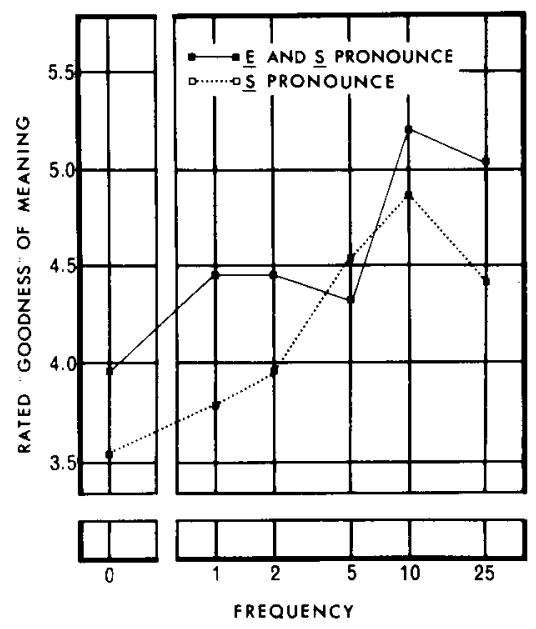

F i g. 1. Average rated "goodness"-of-meaning of nonsense words as a function of frequency of exposure. 